\title{
Absence of depth processing in the large-frame rod-and-frame effect
}

\author{
SHELDON M. EBENHOLTZ \\ University of Wisconsin, Madison, Wisconsin \\ and \\ GERALD W. GLASER \\ Rhode Island School of Design, Providence, Rhode Island
}

\begin{abstract}
Attenuation of the rod-and-frame effect (RFE) with depth separation (Gogel \& Newton, 1975) was investigated with the rod and frame in either intersecting or parallel depth planes (PDP). In the former case, in which either the top of the rod or the frame was inclined $45 \mathrm{deg}$ away from the observer, no attenuation was found for frames projecting a retinal angle of 39.2 or $13.5 \mathrm{deg}$. In the PDP paradigm, the rod was optically $60 \mathrm{~cm}$ nearer the observer than was the frame. The depth adjacency effect of Gogel and Newton was replicated, but only for small retinal angles (7.2 and $6.8 \mathrm{deg}$ ) of the frame and for a 15-deg frame tilt, but not for larger retinal angles (39.2 and $12.7 \mathrm{deg}$ ) or for frames tilted at $22 \mathrm{deg}$. The absence of attenuation with depth separation in large frames and its presence in small frames is consistent with the identification of these phenomena with properties of the ambient and focal visual systems, respectively (Leibowitz \& Post, 1982).
\end{abstract}

The rod-and-frame effect (RFE) represents the influence of a large surrounding frame upon the apparent orientation of a rod enclosed within it, the latter appearing to tilt in a direction opposite to that of the frame (Witkin \& Asch, 1948). Recent studies have failed to disclose any significant role for perceived frame size (Ebenholtz, 1977) or for perceived form (Streibel, Barnes, Julness, \& Ebenholtz, 1980), but have provided evidence that retinal size and linear corner elements in the retinal pattern play a determining role. It therefore appears that the extraction of orientation information precedes both size and form-processing stages.

Since the RFE is largely uninfluenced by such global $^{1}$ spatial attributes as size and form, it appeared unlikely that it would be significantly affected by variations in other global perceptual qualities such as the perceived depth between rod and frame. Yet, Gogel and Newton (1975) have shown that, with an upright rod and tilted frame set stereoscopically at 100 and $160 \mathrm{~cm}$, respectively, perceived rod tilt diminished significantly relative to a coplanar condition. Since the display used by Gogel and Newton (1975) projected about $10 \mathrm{deg}$ of arc side ${ }^{-1}$, it is possible that the reduced illusion they obtained belonged to the domain of small-frame phenomena in which the line ${ }^{2}$ and frame produce an orientation contrast illusion

This research was supported in part by NIH Grant EY02264. S. M. Ebenholtz's mailing address is: Department of Psychology, University of Wisconsin, Madison, Wisconsin 53706.
(Goodenough, Ottman, Sigman, Rosso, \& Mertz, 1979; Wenderoth, 1974). These illusions typically are lower in magnitude than the RFE, can be obtained with an upright frame and a tilted line (Goodenough et al., 1979), and are nonmonotonic with respect to frame tilt over the range from 0 to $45 \mathrm{deg}$ (Beh, Wenderoth, \& Purcell, 1971). Along with these differences, small-frame illusions also may be sensitive to depth differences between rod and frame, whereas large-frame effects may be spared from the influence of depth separation.

The latter possibility was examined in the present study, in which two types of depth separation between rod and frame were investigated in both large and small frames. The two types of separation were produced by maintaining the rod and frame either in parallel (PDP) or intersecting depth planes (IDP). These and the coplanar variations are represented below in Figures 1a, 1b, 1c, 1d, and 1e.

\section{GENERAL PROCEDURE}

\section{Method}

In all experiments, subjects adjusted a luminous line to match their criterion of the egocentric upright. This was defined for the subjects in terms of the chin-to-forehead axis and the 6 to 12 o'clock direction. Two adjustments were made from starting positions at $25 \mathrm{deg} \mathrm{CW}$ and $\mathrm{CCW}$ of true vertical, and bracketing was permitted. After completing line adjustments, the subjects provided estimates of the depth and inclination angle of line and frame. In the PDP studies, the subjects were placed in front of a blank wall, given a foot rule to hold vertically, and asked to place the ruler at a distance from the wall that corresponded to the ap- 

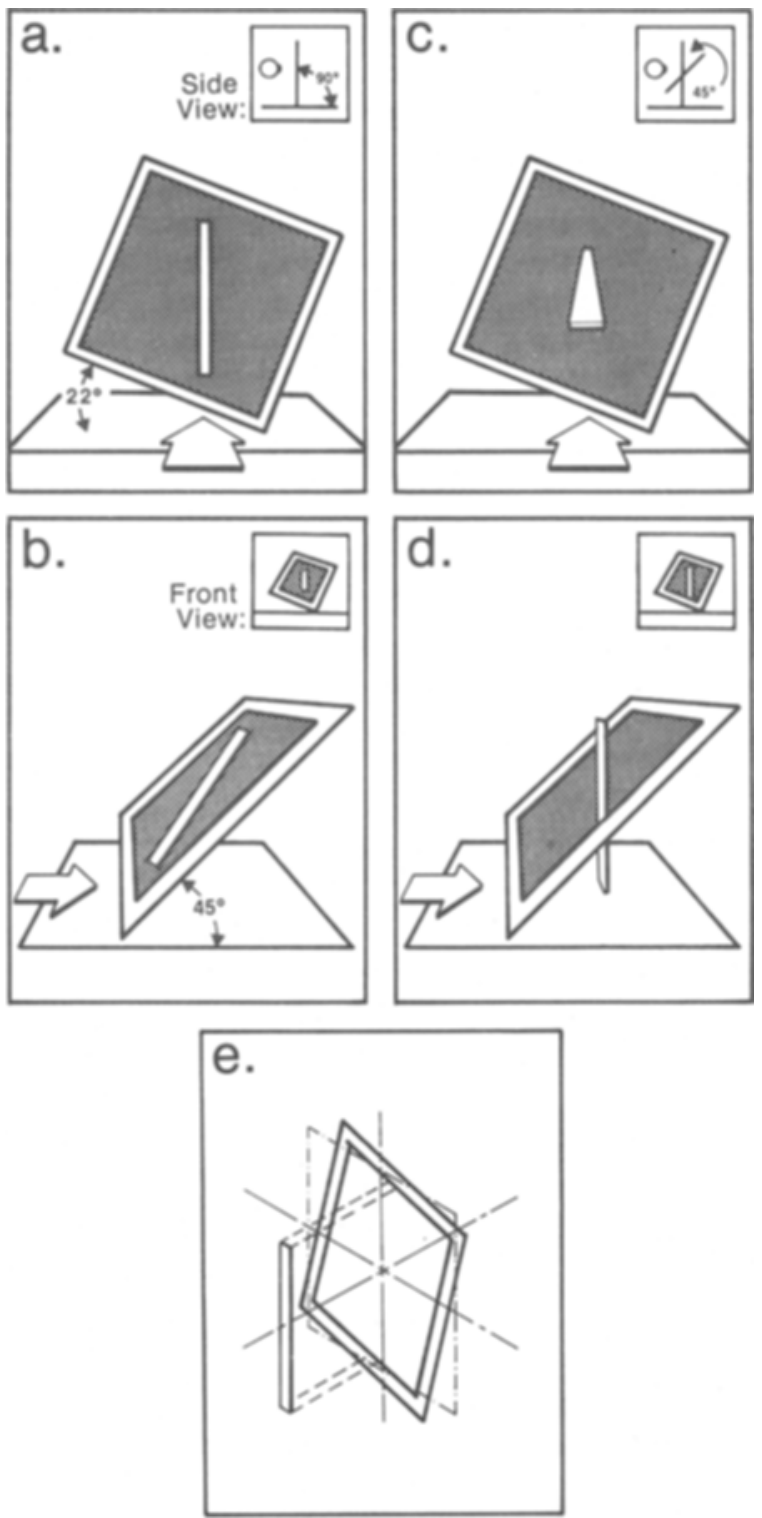

Figure 1. All frames have a 22-deg $\mathrm{CW}$ rotation. Line and frame are both in the observer's frontal plane in 1a, while both are inclined top away by $45 \mathrm{deg}$ in $1 \mathrm{~b}$. The frame is in the frontal plane, but line is inclined 45 deg top away in 1c, while the relations between line and frame are reversed in 1d. Parallel depth planes between line and frame are shown in $1 e$.

parent distance between rod and frame. The subject's setting was then recorded. In the IDP studies, the inclination angle from the frontal plane was estimated by having the subjects retrospectively consider the rod-and-frame display. A ruler was then rotated in depth (top back), and the resultant angle was recorded with the aid of an inclinometer. Subjects who saw no depth difference in the depth (D) relative to the coplanar (C) conditions were replaced, but these amounted to only 6 of 194 subjects. The rod-andframe effect (RFE) was operationally defined as the algebraic difference in mean settings made in the context of a tilted frame and again in the absence of the frame. The frame-absent condition preceded the frame-present condition. In two studies (Experiments 10 and 13, as described below), only the frame-present condition was employed.

\section{Apparatus}

Three rod-and-frame displays of large, medium, and small size were used. These had frame dimensions of $106.8,35.6$, and $18.9 \mathrm{~cm}$ per side, with rod lengths roughly proportional to the frame sizes at $95.3,28.4$, and $16.2 \mathrm{~cm}$, respectively. The displays were made of electroluminescent panels with widths of $2.4,1.2$, and $.5 \mathrm{~cm}$ in the large, medium, and small frames. The respective rod widths were $2.4, .3$, and $.3 \mathrm{~cm}$. Luminance was approximately $1 \mathrm{~cd} \mathrm{~m}^{-2}$.

In all conditions, line and frame were coaxial, rotating about an axis at $121.9 \mathrm{~cm}$ above the floor. Observations were made with the head constrained in a chin- and foreheadrest with the outer canthus of each subject adjusted to the height of the rotation axis of the frame.

In the PDP studies, a depth relation between rod and frame was introduced by optically placing the rod $60 \mathrm{~cm}$ in front of the frame. This was equivalent to the maximum depth separation examined by Gogel and Newton (1975). Figure 2 shows the relative positions of rod and frame in the PDP studies. In these experiments, the line target presented in the depth condition was masked to produce a retinal angle identical to that produced by the line used in the coplanar condition.

In the IDP experiments, depth was introduced either by rotating the top of the rod or the frame, away from the subject. In the latter case, this was in addition to its customary clockwise rotation. In the former case, the rod rotated within its inclined plane while subjects directed the $\mathrm{CW}$ and $\mathrm{CCW}$ adjustments to match the apparent egocentric upright. In none of the present IDP studies was the attempt made to equate the retinal projections of line and/or frame in the inclined and frontal plane orientations.

\section{Design}

The studies compared performance of the same subject under a coplanar $(C)$ condition in which line and frame were in the same

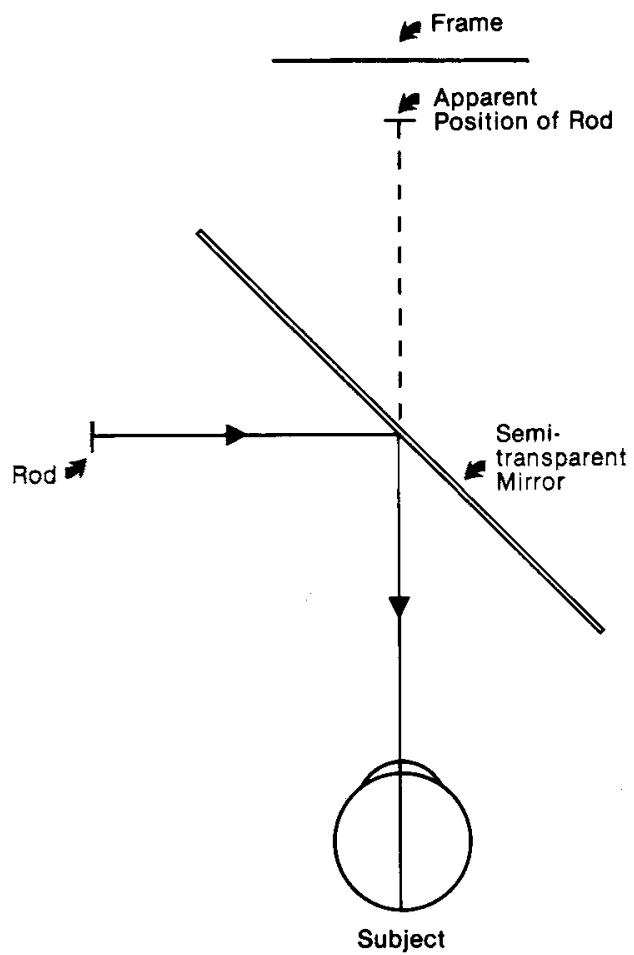

Figure 2. Top view of target and subject positions in PDP studies. 
spatial plane with that in a depth (D) condition in which line and frame occupied different depth planes. The order of testing under $D$ and $C$ conditions was counterbalanced so that for half the subjects in each condition $C$ occurred first and for the remaining subjects $D$ was presented first. In some experiments there were unequal numbers of male and female subjects. However, in all cases gender was balanced across each C-D and D-C sequence so that the two sequences contained equal proportions of each sex.

The subjects were volunteers who had not previously taken part in rod-and-frame studies and participated in no more than one of the experiments reported below.

\section{INTERSECTING DEPTH PLANES (IDP)}

\section{Specific Conditions}

Experiment 1. Six male, 10 female subjects took part. Frame tilt was $22 \mathrm{deg} C W$ at an inclination of $45 \mathrm{deg}$ in the depth condition. The line was always rotated in the frontal plane. The large rod and frame were viewed at a distance of $150 \mathrm{~cm}$, one side of the frame projecting a visual angle of $39.2 \mathrm{deg}$.

Experiment 2. The conditions were the same as in Experiment 1, including the numbers and gender of subjects. In Experiment 2, however, in the depth condition, the rod was inclined $45 \mathrm{deg}$ and the frame remained in the frontal plane.

Experiment 3. The conditions were the same as above except that there were two coplanar conditions: one in which rod and frame both were in the frontal plane and a second in which both were inclined at $45 \mathrm{deg}$. This experiment constituted a control for the possible role of the inclination itself, apart from the depth separation between rod and frame.

Experiment 4. Four male and four female subjects took part. As in Experiment 1, depth was produced by inclining the frame 45 deg relative to the rod. However, there were two differences. First, the medium frame was viewed at $150 \mathrm{~cm}$ to project a retinal angle of $13.5 \mathrm{deg}$ per side. Second, the frame was tilted $15 \mathrm{deg}$ CW. Both variations were undertaken in an attempt to enhance the probability of finding a depth separation effect.

\section{Results}

Table 1 shows the mean difference in rod settings taken without the frame and again in the context of the frame, under coplanar and depth conditions. In all experiments, the coplanar-depth sequence never proved to be significant as tested by the Grant (1947) test. Consequently, data of both sequences were combined in the coplanar-depth comparisons and the two coplanar comparisons of Experiment 3. In the latter case the comparison of the two coplanar conditions showed no significant differences; that is, whether both rod and frame were inclined or both were in the frontal plane was not a determining factor in the RFE, which in all cases was significantly greater than zero at $\mathrm{p}=.05$ or better. Thus, inclination by itself, at least to $45 \mathrm{deg}$, should not be expected to influence the RFE, which is thus shown to be remarkably robust.

Experiments 1 and 2 both showed no significant differences in RFE between the depth and the coplanar conditions. Thus, for a $22-\mathrm{deg} \mathrm{CW}$ frame tilt with a large retinal angle, neither rod nor frame inclination was effective in reducing the RFE. The same pattern of equivalence in coplanar and inclined plane conditions also can be extended to the mediumsized inclined frame at a 15-deg CW tilt. This is shown in the data of Experiment 4. That the absence of any depth separation effect is not due to a failure of subjects to appreciate the depth interval is shown in the right-hand column of Table 1. Here the subject's estimates of the angular inclination of rod and frame leave no doubt that the depth was experienced, although it was underestimated with frame inclination and estimated quite veridically with rod inclination. ${ }^{3}$

The IDP studies show that for frames as small as 13.5 deg in retinal angle, separation in oblique depth planes is insufficient to reduce the RFE. It is possible that yet smaller retinal angles would have sufficed, but these were not investigated further in the IDP paradigm.

\section{PARALLEL DEPTH PLANES (PDP)}

\section{Experiments 5-7 investigated 22-deg $\mathrm{CW}$ frame} tilts, while Experiments 8-13 examined 15-deg CW tilts.

\section{Specific Conditions}

Experiment 5. Six male and six female subjects took part. The large frame produced a retinal angle of $39.2 \mathrm{deg}$ at $150 \mathrm{~cm}$. In the depth condition, the line was set optically at $90 \mathrm{~cm}$, yielding a 60 -cm depth interval and a binocular disparity of about $1.66 \mathrm{deg}$. Frame tilt was $22 \mathrm{deg} \mathrm{CW}$.

Experiment 6. Six female and two male subjects viewed the small frame with a retinal angle of $7.2 \mathrm{deg}$. Other conditions were the same as those of Experiment 5.

Experiment 7. Eight male and eight female subjects viewed the small frame replicating the conditions of Experiment 6 . The only

Table 1

Intersecting Depth Planes: Means and Standard Errors of the Rod(R)-and-Frame(F) Effect for Coplanar (C) and Inclined-Planes (I) Conditions

\begin{tabular}{|c|c|c|c|c|c|c|c|c|c|c|c|c|c|c|}
\hline \multirow{2}{*}{$\begin{array}{l}\text { Exper- } \\
\text { iment }\end{array}$} & \multirow[b]{2}{*}{$\mathrm{n}$} & \multirow[b]{2}{*}{$\mathbf{r}_{\mathrm{S}}$} & \multirow{2}{*}{$\begin{array}{c}\text { Frame } \\
\text { Tilt }\end{array}$} & \multirow{2}{*}{$\begin{array}{l}\text { Visual } \\
\text { Angle }\end{array}$} & \multicolumn{2}{|c|}{ C } & \multicolumn{2}{|c|}{ I } & \multirow[b]{2}{*}{$\mathrm{I}<\mathrm{C}$} & \multicolumn{2}{|c|}{$C-I$} & \multirow{2}{*}{$\begin{array}{c}\text { Actual } \\
\text { Inclination }\end{array}$} & \multicolumn{2}{|c|}{$\begin{array}{c}\text { Inclination } \\
\text { Estimate }\end{array}$} \\
\hline & & & & & Mean & SE & Mean & SE & & Mean $_{\mathrm{d}}$ & $\mathrm{SE}_{\mathrm{d}}$ & & Mean & SE \\
\hline 1 & 16 & .65 & $22 \mathrm{CW}$ & 39.2 & 6.59 & 1.09 & 7.14 & .86 & $6 / 16$ & -.55 & .84 & $45(F)$ & 33.44 & 3.09 \\
\hline 2 & 16 & .87 & $22 \mathrm{CW}$ & 39.2 & 5.11 & .93 & 5.17 & .95 & $7 / 16$ & -.06 & .48 & $45(\mathrm{R})$ & 45.94 & 2.34 \\
\hline 3 & 16 & .84 & $22 \mathrm{CW}$ & 39.2 & 4.94 & .76 & 4.31 & .86 & $8 / 16$ & .63 & .47 & $45(\mathrm{R}+\mathrm{F})$ & 36.25 & 2.72 \\
\hline 4 & 8 & .89 & $15 \mathrm{CW}$ & 13.5 & 3.05 & 1.00 & 3.01 & 1.03 & $6 / 8$ & .04 & .47 & $45(F)$ & 35.63 & 4.06 \\
\hline
\end{tabular}

Note $-r_{s}=$ correlation between coplanar and inclined $R F E ; I<C=$ proportion of subjects showing a lower $R F E$ in $I$ relative to $C$. Frame tilt, visual angle, and inclination are given in degreps. For Experiment 3 , the estimate of frame inclination is given; rod estimate was $35.00 \mathrm{deg}(S E=2.66)$. $C=$ coplanar $;=$ inclined. 
difference was that subjects were asked to describe the displays in the coplanar and depth (frame-present) conditions prior to making line adjustments. This served to direct attention to the depth interval.

Experiment 8. Six female and six male subjects viewed the large frame at $150 \mathrm{~cm}$. Conditions were the same as those of Experiment 5 except that the frame tilt was $15 \mathrm{deg} \mathrm{CW}$.

Experiment 9. Six male and 10 female subjects viewed the medium-sized frame at $160 \mathrm{~cm}$, producing a retinal angle of $12.7 \mathrm{deg}$. In the depth condition, the line was placed at $100 \mathrm{~cm}$, yielding a $60-\mathrm{cm}$ depth interval with a binocular disparity of about $1.39 \mathrm{deg}$. Frame tilt was $15 \mathrm{deg} \mathrm{CW}$.

Experiment 10. Six male and six female subjects were exposed to conditions that replicated those of Experiment 9 with one exception. In Experiment 10, the line-alone condition that normally preceded the line and frame presentation was omitted. Hence, no baseline or bias estimate was available. In this case, the RFE was calculated directly from mean settings with frame present. In this respect, the conditions duplicated those used by Gogel and Newton (1975).

Experiment 11. Eight male and eight female subjects were exposed to the small frame at $160 \mathrm{~cm}$, thereby producing a retinal angle of $6.8 \mathrm{deg}$. The line in the depth condition was at $100 \mathrm{~cm}$, and frame tilt was $15 \mathrm{deg} \mathrm{CW}$. Special instructions were to ignore the frame and fixate the rod under both coplanar and depth conditions. The intention was to reduce the possibility of a depth separation effect attributable to a shift in attention from frame to rod in the depth condition. ${ }^{4}$

Experiment 12. All conditions were the same as those of Experiment 11 except that the frame was viewed at $150 \mathrm{~cm}$, producing a retinal angle of $7.2 \mathrm{deg}$, while the line was placed at $90 \mathrm{~cm}$ in the depth condition. Thus, binocular disparity was somewhat enhanced over that of Experiment 11.

Experiment 13. Six male and six female subjects viewed the small frame at $150 \mathrm{~cm}$ with a $15-\mathrm{deg} \mathrm{CW}$ tilt. Conditions were the same as in Experiment 12, except that no special fixation instructions were used and there was no line-alone control setting.

\section{Results}

There were no significant sequence effects; hence, all data were combined over test sequence. Summary data of all nine experiments are shown in Table 2. The rightmost columns show clearly that the depth intervals between rod and frame were always appreciated, although verbal estimates in Experiments 6, 7, and 8 were consistently lower than those made by placing the ruler in front of a blank wall. The third column demonstrates that subjects' performance remained highly correlated in the coplanar and depth conditions. Thus, a strong common component remains despite the radical change produced by the depth interval.

The major results are shown in the columns labeled "C-D," expressing the mean differences between coplanar and depth conditions. No significant reductions in RFE occurred until the frame size fell below $7.2 \mathrm{deg}$ and the frame tilt was $15 \mathrm{deg}$. Only then, as the results of Experiments 11, 12, and 13 show, did the depth separation produce a significant decrement. The column headed " $D<C$ " also supports this conclusion by demonstrating an increase in the number of subjects for whom the depth condition produced a lower RFE relative to the coplanar condition.

A surprising result can be seen in the distribution of the RFE magnitudes in the columns labeled " $C$ " for coplanar and " $D$ " for depth. Because of the results of previous studies (Ebenholtz, 1977; Ebenholtz \& Callan, 1980), a drop in RFE with retinal size of frame was expected, but the present data show this trend most clearly for the 22-deg frame tilt. Here (Experiment 6), with the visual angle of the frame equal to $7.2 \mathrm{deg}$, the RFE actually failed to differ significantly from zero. In contrast, with the 15 -deg frame tilt and a visual angle of $6.8 \mathrm{deg}$ (Experiment 11), a significant 3-deg RFE was obtained. The resulting trends are apparent in Figure 3, which represents the PDP data pooled over common retinal angles. ${ }^{5}$ Retinal size is a much more effective variable with a 22-deg frame tilt than with one of $15 \mathrm{deg}$. Alternately, it may be noted that at large retinal angles there is an increase in RFE with increasing frame tilt from 15 to $22 \mathrm{deg}$, whereas at small retinal angles increasing frame tilt produces a drop in RFE. Of course, these results could be due to differences between individuals forming the groups, but there also are valid theoretical and empirical

Table 2

Parallel Depth Planes: Means and Standard Errors of Rod and Frame Effect for Coplanar (C) and Depth (D) Conditions

\begin{tabular}{|c|c|c|c|c|c|c|c|c|c|c|c|c|c|c|}
\hline \multirow{2}{*}{$\begin{array}{c}\text { Exper- } \\
\text { iment }\end{array}$} & \multirow[b]{2}{*}{$\mathbf{n}$} & \multirow{2}{*}{$\begin{array}{c}\text { Frame } \\
\mathrm{I}_{\mathrm{S}}\end{array}$} & \multirow{2}{*}{$\begin{array}{c}\text { Visual } \\
\text { Tilt }\end{array}$} & \multirow[b]{2}{*}{ Angle } & \multicolumn{2}{|c|}{$\mathrm{C}$} & \multicolumn{2}{|c|}{ D } & \multirow[b]{2}{*}{$\mathrm{D}<\mathrm{C}$} & \multicolumn{2}{|c|}{$C-D$} & \multirow{2}{*}{$\begin{array}{l}\text { Actual } \\
\text { Depth }\end{array}$} & \multicolumn{2}{|c|}{ Estimated Depth } \\
\hline & & & & & Mean & SE & Mean & $\mathrm{SE}$ & & Mean & $\mathrm{SE}$ & & Mean & SE \\
\hline 5 & 12 & .74 & $22 \mathrm{CW}$ & 39.2 & 6.50 & 1.14 & 7.21 & 1.35 & $5 / 12$ & -.71 & .92 & 60 & 64.88 & 7.30 \\
\hline 6 & 8 & .83 & $22 \mathrm{CW}$ & 7.2 & -.25 & .91 & .31 & .64 & $3 / 8$ & -.56 & .52 & 60 & 20.16 & 4.31 \\
\hline 7 & 16 & .59 & $22 \mathrm{CW}$ & 7.2 & .95 & .46 & 1.06 & .54 & $8 / 16$ & -.11 & .46 & 60 & 34.93 & 5.52 \\
\hline 8 & 12 & .71 & $15 \mathrm{CW}$ & 39.2 & 4.50 & .73 & 5.33 & .75 & $2 / 12$ & -.83 & .56 & 60 & 35.98 & 5.16 \\
\hline 9 & 16 & .68 & $15 \mathrm{CW}$ & 12.7 & 4.37 & .88 & 4.20 & .72 & $9 / 16$ & .17 & .66 & 60 & 49.85 & 4.48 \\
\hline 10 & 12 & .77 & $15 \mathrm{CW}$ & 12.7 & 4.02 & .58 & 4.44 & .81 & $6 / 12$ & -.42 & .52 & 60 & 71.54 & 7.46 \\
\hline 11 & 16 & .81 & $15 \mathrm{CW}$ & 6.8 & 3.03 & .47 & 2.42 & .55 & $12 / 16$ & $.61 *$ & .33 & 60 & 63.82 & 9.69 \\
\hline 12 & 16 & .74 & $15 \mathrm{CW}$ & 7.2 & 3.98 & .50 & 3.05 & .42 & $13 / 16$ & $.93^{*}$ & .34 & 60 & 61.28 & 8.83 \\
\hline 13 & 12 & .84 & $15 \mathrm{CW}$ & 7.2 & 4.46 & .98 & 3.02 & .77 & $9 / 12$ & $1.44^{*}$ & .53 & 60 & 58.21 & 3.24 \\
\hline
\end{tabular}

Note- $r_{s}=$ correlation between $R F E$ s in coplanar and depth conditions; $D<C=$ proportion of subjects showing a lower $R F E$ in $D$ relative to $C$. Frame tilt and visual angle are given in degrees. Depth estimates, given in centimeters, were verbal from memory in Experiments 6, 7, and 8. $C=$ coplanar; $D=$ depth. $\quad *$ Significant at $p<.05$. 


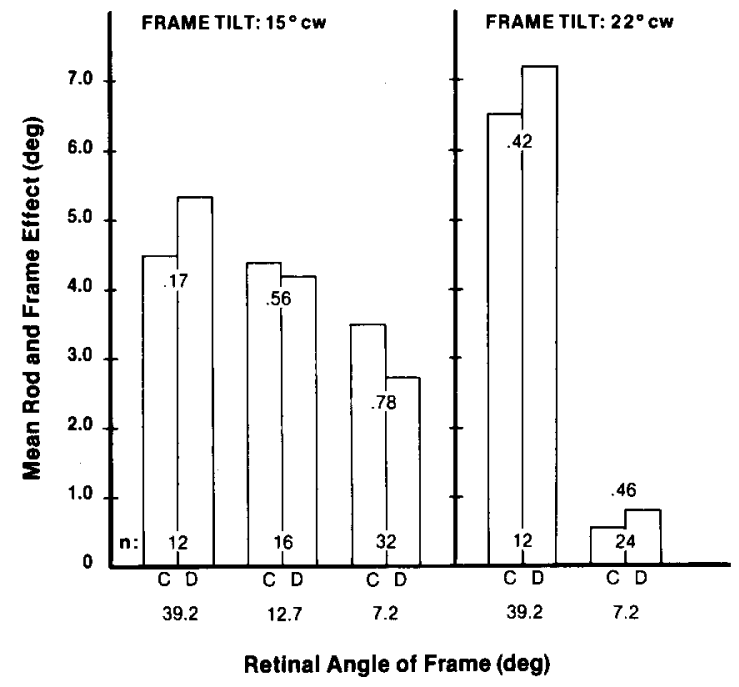

Figure 3. Mean RFE under coplanar (C) and depth (D) conditions by frame tilt (15 and $22 \mathrm{deg} \mathrm{CW}$ ) and retinal angle. Proportions represent relative numbers of subjects for whom the RFE was less in D than in C.

reasons for believing that the RFE interacts both with the visual angle of the frame and the magnitude of frame tilt. These will be taken up below.

\section{DISCUSSION}

For large frames, probably beyond about $10 \mathrm{deg}$ of visual angle, a readily apparent depth separation between rod and frame in the PDP configuration was without influence on the RFE. Frames with smaller retinal angles of 6.8 and 7.2 deg of arc with a 15-deg tilt did, however, exhibit the reduction with depth separation, and thus the results of Gogel and Newton (1975) were replicated. The adjacency effect was relatively low in magnitude (under $2 \mathrm{deg}$ ), but it is robust in that we obtained the effect with the method of adjustment whereas Gogel and Newton employed a kinesthetic matching technique. With the rod and frame in intersecting planes inclined at $45 \mathrm{deg}$, frames as small as $13.5 \mathrm{deg}$ in visual angle showed no reduction in the RFE. These results indicate that the influence of depth adjacency is limited to small frames represented, approximately, in parafoveal regions. ${ }^{6}$ Gogel's adjacency principle (Gogel, 1978) could accommodate this limitation on the premise that relative tilt cues play a predominant role in small frames whereas absolute tilt cues (i.e., egocentric orientation) govern apparent rod orientation in large frames. One problem for this approach is the absence of some a priori method to assess the relative weight of each cue. Thus, for example, prior to the present study there was no basis for predicting that relative cues would play no role at all in the large-frame conditions, in which depth separation was without ef- fect, but that these cues would dominate orientation perception in small frames.

The failure of large frames to exhibit the depth separation effect is consistent with previous experiments showing the RFE to be essentially uninfluenced by processing stages associated with global perceptual properties such as size and shape. The present results show that depth processing can be added to this list. The emerging view of the (large-frame) RFE is that of an automatic reflex-like phenomenon controlled by local retinal stimulation, influencing visual egocentric orientation (Ebenholtz \& Callan, 1980).

Two interaction effects, possibly related, are suggested by the data, as shown in Figure 3. First, it is clear that depth separation effectively reduced the RFE at small retinal angles, but only with a 15-deg frame tilt. No reduction with depth separation was found at a frame tilt of $22 \mathrm{deg}$. Second, considering only the RFE obtained under coplanar conditions, increasing frame tilt from 15 to $22 \mathrm{deg}$ increased the magnitude of the RFE for large retinal angles but reduced the RFE for small retinal angles. These results suggest important differences in the nature of small- and large-frame effects.

Several recent studies using small frames may shed particular light on these results. For example, Wenderoth (1974), using a line-alone control condition with the method of adjustment as in the present studies, found that a frame projecting a retinal angle of $6.47 \mathrm{deg}$ yielded very small effects, no higher than about 1 deg. Furthermore, the obtained errors initially were positive in the direction of the frame, and then decreased with increasing frame tilt from 15 to $25 \mathrm{deg} \mathrm{CW}$. The function then became negative, yielding errors opposite to frame tilt, finally reversing and reaching zero again at a 45-deg frame tilt. A similar function of frame tilt had been obtained by Beh et al. (1971) but without line-alone control measures. In that study, the frame projected $7.63 \mathrm{deg}$ side $^{-1}$ and illusion magnitudes were also quite low, averaging under $1.5 \mathrm{deg}$. In both studies, a frame tilt of $15 \mathrm{deg}$ produced significant positive errors but frame tilts in the vicinity of $25 \mathrm{deg}$ were low and not significantly different from zero. Thus, for small frames, the differences in RFE obtained in the present study between the 15- and 22-deg frame-tilt conditions are quite consistent with the angular function of Beh et al. (1971) and Wenderoth (1974). But why should there be no adjacency effect, that is, no reduction in the RFE for 22-deg frame tilts with depth separation? Two reasons seem likely. First, the low magnitudes of effect in the 22-deg coplanar condition make it statistically unlikely that a further reduction under the depth condition could be shown to be statistically significant. Second, even if it is assumed that depth separation reduces the effect, perhaps by inhibiting the angle illusion between rod and frame edge (Goodenough et al., 1979; Wenderoth, 1974), 
since there is essentially no illusion at $22 \mathrm{deg}$, in principle there could be no measurable departure from it in the presence of a depth separation.

We have offered an explanation of our small-frame results in terms of the nonmonotonic properties of the small-frame angular function (Beh et al., 1971). A similar explanation may be adduced for the obtained increase in RFE in large frames with increases in frame tilt from 15 to $22 \mathrm{deg}$. This would be possible if it were true that the large-frame angular functions were monotonic within the range of frame tilts investigated. There appear to be only two large-frame studies available on the RFE as a function of frame tilt (Graf, 1966; Lichtenstein \& Saucer, 1972). In Graf's studies, the frame projected a retinal angle of $40.3 \mathrm{deg}$, whereas the frame used by Lichtenstein and Saucer was rectangular and yielded a retinal projection of $29.5 \times 35.1 \mathrm{deg}$ of arc. In both studies, frame tilt was varied from upright to 45 deg of tilt. Graf's study showed a monotonic increase from 0 to $30 \mathrm{deg}$ of frame tilt with a drop at $45 \mathrm{deg}$, while Lichtenstein and Saucer's data showed an approximately linear function increasing from 0 to $20 \mathrm{deg}$ of frame tilt with some evidence for a leveling off or a drop thereafter, depending upon the individual subject. In neither case did the functions become negative with increasing frame tilt. The present results, showing an increase in RFE between 15 and $22 \mathrm{deg}$ of frame tilt, are thus quite consistent with the positive monotonicity of the large-frame angular function. ${ }^{7}$

The present studies thus contribute further evidence to the conclusion that large- and small-frame effects are quite different phenomena, reflecting the functional differences inherent in the ambient and focal visual systems, respectively (Leibowitz \& Post, 1982; Schneider, 1969). The RFE would appear to be a paradigm case of ambient system functioning. By providing a reference signal for egocentric orientation perception and the control of body posture (Ebenholtz \& Benzschawel, 1977; Sigman, Goodenough, \& Flannagan, 1979; Lee \& Aronson, 1974), eccentrically located retinal patterns serve the function of an artificial horizon or a peripheral visual orientation guidance system. As such, it is appropriate that they act automatically without the necessity of prior perceptual or cognitive processing for such attributes as size, shape, and depth. ${ }^{8}$ The latter, on the other hand, require focal system processing in form recognition and identification of specific pattern features. The identification of angle magnitude between line elements would certainly seem to be such a focal task, and, in fact, an angle illusion of several degrees has been implicated in the RFE (Goodenough et al., 1979). If, indeed, the smallframe illusion results from processing within the focal system, then it is reasonable to expect the output of these processing stages to play a role in the illusion. ${ }^{9}$ The RFE, on the other hand, can be con- strued as a direct response to eccentrically located retinal patterns, rather than as a secondary process based upon information for size, shape, and depth.

\section{REFERENCES}

Beh, H. C., Wende roth, P. M., \& Purcell, A. T. The angular function of a rod-and-frame illusion. Perception \& Psychophysics, 1971, 9, 353-355.

Ebenholtz, S. M. Determinants of the rod and frame effect: The role of retinal size. Perception \& Psychophysics, 1977, 22, 531-538.

Ebenholtz, S. M., \& Benzschawel, T. L. The rod and frame effect and induced head tilt as a function of observation distance. Perception \& Psychophysics, 1977, 22, 491-496.

Ebenholtz, S. M., \& Callan, J. W. Modulation of the rod and frame effects: Retinal angle vs. apparent size. Psychological Research, 1980, 42, 327-334.

Fox, R., \& PAtTerson, R. Depth separation and lateral interference. Perception \& Psychophysics, 1981, 30, 513-520.

GoGEL, W. C. The adjacency principle in visual perception. Scientific American, 1978, 238, 126-139.

Gogel, W. C., \& Newton, R. E. Depth adjacency and the rodand-frame illusion. Perception \& Psychophysics, 1975, 18, 163-171.

Goodenough, D. R., Oltman, P. K., Sigman, E., Rosso, J., \& MERTZ, H. Orientation contrast effects in the rod-and-frame test. Perception \& Psychophysics, 1979, 25, 419-424.

GRAF, R. G. The relationship between perceived head position and the perception of the vertical. Unpublished MA thesis, Connecticut College, 1966.

Grant, D. A. The statistical analysis of a frequent experimental design. American Journal of Psychology, 1949, 62, 119-122.

JULESZ, B. Global stereopsis: Cooperative phenomena in stereoscopic depth perception. In R. Held, H. W. Leibowitz, \& H. -L. Teuber (Eds.), Handbook of sensory physiology (Vol. 8): Perception. Heidelberg: Springer-Verlag, 1978.

LEE, D., \& Aronson, E. Visual proprioceptive control of standing in human subjects. Perception \& Psychophysics, 1974, 15, 529-532.

Leнмкuнle, S. W., \& Fox, R. The effect of depth separation on metacontrast masking. Journal of Experimental Psychology: Human Perception and Performance, 1980, 6, 605-621.

Leibowitz, H. W., \& Post, R. B. The two modes of processing concept and some implications. In J. J. Beck (Ed.), Organization and representation in perception, Hillsdale, N. J: Erlbaum, 1982.

Lichte NSTEIN, J. H., \& SAUCER, R. T. Experimental investigation of the visual field dependency in the erect and supine positions (Technical Note D-6883). Hampton, Va: NASA Langley Research Center, 1972 (NTIS, Springfield, Va.).

Ogle, K. N., \& Elle rbrock, V. J. Cyclofusional movements. Archives of Ophthalmology, 1946, 36, 700-735.

SchNeIDER, G. E. Contrasting visuometer functions of tectum and cortex in the golden hamster. Psychologische Forschung, $1969,31,52-62$.

Sigman, E., Goodenough, D. R., \& Flannagan, M. Instructions, illusory self-tilt and the rod-and-frame test. Quarterly Journal of Experimental Psychology, 1979, 31, 155-165.

Streibel, M. J., Barnes, R. D., Julness, G. D., \& Ebenholtz, S. M. Determinants of the rod and frame effect: Role of organization and subjective contour. Perception \& Psychophysics, $1980,27,136-140$.

Wenderoth, P. M. The distinction between the rod-and-frame illusion and the rod-and-frame test. Perception, 1974, 3, 205-212.

WitкıN, H. A., \& Asch, S. E. Studies in space orientation. IV. Further experiments on perception of the upright with displaced visual fields. Journal of Experimental Psychology, 1948, 38, 762-782. 


\section{NOTES}

1. Size, form, and depth are regarded as gestalt, or global, qualities in the sense suggested by Julesz (1978) that local retinal stimulation alone is inadequate to account for them. Rather, some further organizing or processing stages are required. Although stereopsis from monocular contours may be guided by local retinal disparities, the orderly distribution in space of the apparent depth intervals that correspond to these disparities also reflects the operation of an organizing stage. Thus, even locally determined stereopsis requires global processing. In any event, form processing is thought to play a role in guiding stereopsis with monocular contours. Hence, depth could not precede the form-processing stage.

2. The terms "line" and "rod" are used interchangeably.

3. This probably reflects a slight ocular extortion (Ogle \& Ellerbrock, 1946) induced by the inclined frames. This, in turn, would reduce the apparent inclination of the frame and the rod as well when both were present and coplanar, as in Experiment 3. Since the inclined rod probably is not as adequate a stimulus to cyclovergence as is the frame, veridical judgments of the rod are more likely. Estimates of the inclination of the upright rod and frame were, with two minor exceptions, veridical.

4. We thank Professor Gogel for raising this possibility. Pilot observations with the large frame at $150 \mathrm{~cm}$ showed no effects of instructions to ignore the frame on the RFE, under depth and coplanar conditions.

5. Data of 6.8- and 7.2-deg frame tilts also were pooled. Data of Experiments 10 and 13 were not included because, in these experiments, the RFE was computed directly without reference to a line-alone condition.

6. It is not known whether, in contrast with large frames, changes in apparent size with constant retinal size also influence the magnitude of small-frame illusions.

7. It would seem to be worthwhile to continue the practice (Gogel \& Newton, 1975; Wenderoth, 1974) of referring to smallframe phenomena as "illusions," or RFI, and large-frame phenomena as "effects," or RFE (Ebenholtz, 1977).

8. In the case of random-dot stereograms, depth processing may indeed be preliminary to form processing (e.g., Fox \& Patterson, 1981; Lehmkuhle \& Fox, 1980), but these are differentiations made within the focal systems.

9. Accordingly, the domain of the adjacency principle may be constrained to focal system phenomena.

(Manuscript received December 3, 1981; revision accepted for publication April 13, 1982.) 